\title{
Features of mathematical modelling of the microclimate of the non-productive building
}

\author{
Maria Prorokova ${ }^{1, *}$, Vyacheslav Bukhmirov ${ }^{1}$ \\ ${ }^{1}$ Ivanovo State Power University named after V.I. Lenin, 153003 Ivanovo, Russia
}

\begin{abstract}
The mathematical model of heat and mass transfer in a nonproduction building is presented in the article. Features of modeling of objects of the specified type are analyzed. A mathematical model of the microclimate in the ANSYS Fluent was developed. Verification of the model was carried out by comparing the values of the microclimate parameters obtained by calculation, with experimental data.
\end{abstract}

\section{Introduction}

Calculation of the main parameters of the microclimate in non-production buildings is done to determine the degree of comfort of the microclimate, so the calculation must be carried out both during construction and when the building structure is changed, including for energy saving during its operation (replacement of windows, thermal insulation a. o.). All calculations of microclimate parameters can be conditionally divided into two groups. The first group includes, so-called, engineering calculations, which are based on integral or balance models. Balance models are also called models with lumped parameters or zerodimensional mathematical models. Balance models analyze the room as a whole, in which the temperature and humidity of the air are the same at all points in the room. All engineering calculations are based on the solution of the equations of thermal and material balances for the object, and the boundary conditions are set, as a rule, using the equations of stationary heat transfer through non-permeable walls.

Due to increase in power of computing tools and improving their mathematical apparatus, including numerical methods for solving differential heat transfer, mass, and momentum transfer equations, it became possible to predict the microclimate in rooms using multidimensional non-stationary mathematical models. This group of calculation methods is traditionally called mathematical modeling of microclimate [1].

For the numerical solution of the system of transport equations for the main parameters of a substance (heat, mass, and momentum), so-called, software-computational complexes (SCC) are developed, which can be universal or adapted for modeling physical and chemical processes in a specific device, for example, in a nuclear reactor. In this work the simulation of the microclimate in a non-production room was performed in the SCC ANSYS Fluent, which refers to the universal SCC, which allows modeling the features of physical and chemical processes in various objects. When using ANSYS Fluent is very

\footnotetext{
* Corresponding author: prorokova mv@list.ru
} 
important is the stage of mathematical formulation of the problem of calculating a particular process. At the stage of the mathematical description of the object, the assumptions that simplify the problem write, define the conditions for single-valuedness, and write the differential transfer equations.

\section{Features of mathematical modeling of microclimate for non- production objects}

In non-production premises, the microclimate is formed by convective, conductive and radiative heat transfer, and also by molecular and convective diffusion of air pollutants. Consequently, the mathematical model for the formation of a microclimate in a buildings room must include the energy transfer equations due to radiative and convective heat transfer, the momentum transfer equation, the continuity equation, and the mass transfer equation for each component of the mixture, taking into account the assumptions: air in the room is a four-component mixture, that consists of nitrogen, oxygen, carbon dioxide and water vapor; the components of the gas mixture and the mixture itself obey the laws of an ideal gas; in the room there is a thermo gravitational movement of air; gas in the room is a non-diathermic substance; diffusion transport of air through the solid elements of the enclosing structures (walls, linen of the window block) is absent; the airflow inlet is through the window, the outlet is through the ventilation openings and doors; sources of heat due to internal friction are small; scattering and refraction of radiant energy are not taken into account.

The mathematical model of heat and mass transfer in the building of a non-production building looks as follows:

$$
\begin{gathered}
\frac{\partial}{\partial t} \rho E+\nabla \overrightarrow{u p} E=\mathrm{V}\left(\lambda_{e f f} \mathrm{~V} T-\sum_{i=1}^{n} h_{i} \overrightarrow{J_{i}}\right)+q_{v} . \\
\frac{d I(\vec{r}, \vec{s})}{d s}+\left(A+\sigma_{s}\right) I(\vec{r}, \vec{s})=A \frac{\sigma_{0} T^{4}}{\pi}+\frac{\sigma_{s}}{4 \pi} \int_{0}^{4 \pi} I\left(\vec{r}, \overrightarrow{s^{\prime}}\right) d \Omega^{\prime} . \\
\frac{\partial}{\partial t} \rho+\nabla(\rho \vec{w})=0 ; \\
\frac{\partial}{\partial t} \rho \vec{w}+\nabla(\rho \vec{w} \vec{w})=\nabla \rho v \nabla \vec{w}+\rho \vec{g} ; \\
\frac{\partial}{\partial t} \rho C_{i}+\nabla\left(\rho \vec{w} C_{i}\right)=\nabla\left(\left(\rho D_{i, m}+\rho D_{t}\right) \nabla C_{i}\right)+S_{i} .
\end{gathered}
$$

In equations (1) - (5): $t$ - time; $\rho$ - density of the gas mixture; $\vec{w}$ - speed of air movement; $E$ - specific internal energy of the gas mixture; $\lambda_{\text {eff }}$ - effective coefficient of thermal conductivity; $h_{i}$ - specific enthalpy of the i-th component of the mixture; $\vec{r}-$ radius-vector; $\vec{s}$ - unit vector in the direction of scattering; $\overrightarrow{s^{\prime}}-$ unit vector in the direction of the incident radiation; $A$ - absorption coefficient; $\sigma_{\mathrm{s}}-$ scattering coefficient (for pure air $\left.\sigma_{\mathrm{s}}=0\right) ; \sigma_{0}$ - Stefan-Boltzmann constant; $I$ - brightness of radiation at a given point $(\vec{r})$ in a given direction $(\vec{s}) ; T-$ local temperature; $\Omega$ - solid angle; $q_{v}$ - capacity of internal sources of heat; $v$ - kinematic viscosity coefficient; $g$ - acceleration of gravity; $\overrightarrow{J_{i}}$ 
- density of the diffusion flux of the $i$-th component of the mixture; $C_{i}$ - local mass concentration of the $i$-th component of the mixture; $D_{i, m}$ - diffusion coefficient of the $i$-th component of the mixture; $D_{t}$ - coefficient of turbulent diffusion; $S_{i}$ - mass source of the $i$ th component of the mixture.

A detailed description of the mathematical model is given in $[1,2,3]$.

Thermophysical and radiation properties of objects that participate in the process of heat and mass transfer are determined from reference data [4], and the radiative properties of the air mixture are calculated by specifying the volume fractions of the individual components. Sources of thermal energy, moisture and carbon dioxide in the premises of residential, public and administrative buildings are people, heating appliances and household appliances. It is known that, on average, a person exhales in a second $1,2 \cdot 10^{-4} \mathrm{\kappa} \Gamma$ air, whose composition 78,5\% $\mathrm{N}_{2}, 14,2 \% \mathrm{O}_{2}, 3,6 \% \mathrm{H}_{2} \mathrm{O}, 3,7 \% \mathrm{CO}_{2}$, and emits $105 \mathrm{Wt}$ of thermal energy [5].

Starting conditions for the solution of the system of equations (1) - (5) (temperature of surfaces of the room, air temperature, the relative humidity and speed and speed of indoor and outdoor air) are determined from the experimental data.

The power of the heating devices is determined experimentally, and the heat dissipation of electrical appliances is set according to the equipment's passport data, depending on the mode of operation of the equipment. Temperature of surfaces of the room $\left(T_{w}\right)$ and sources of thermal energy is accepted by results of the experiment.

The air velocity is calculated from the experimental data or the normative value of the air exchange [6].

The k- $\varepsilon$ model of turbulence was chosen to model turbulence from the analysis of literature [7] and analysis of the results of computational experiments [3, 7].

The geometric and grid models of the experimental room of the university's educational audience in the ANSYS Fluent SCC and the features of the computing process of microclimate parameters are described in detail in the paper [8].

Figure 1 shows the results of mathematical modeling and experimental measurement of air temperature at four points in the experimental room: in the center of the room (point 1 on the figure 1), at a distance of $0.5 \mathrm{~m}$ from the left wall (point 2 on the figure 1) and at a distance of $0.5 \mathrm{~m}$ from the front wall (point 3 on the figure 1).

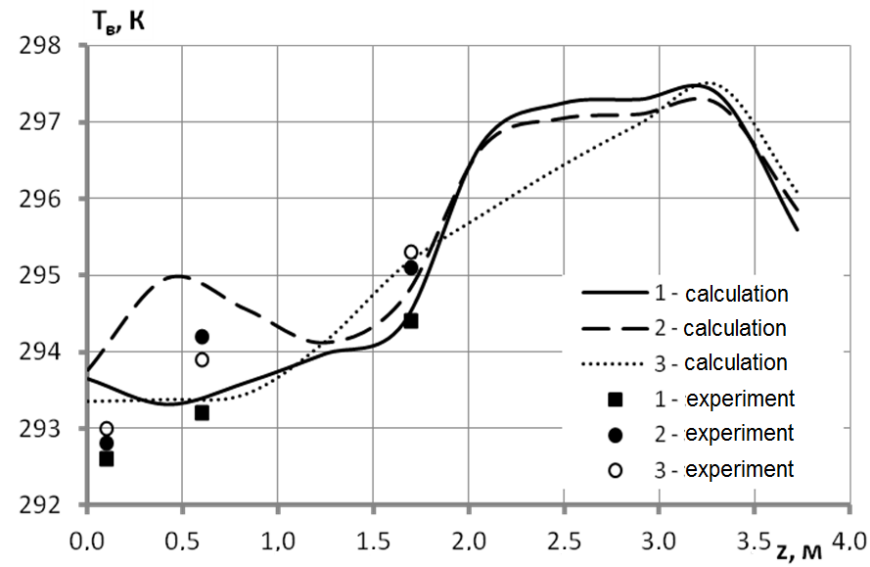

Fig. 1. Air temperature in the experimental room.

\section{Results and discussion}


Analysis of figure 1 shows a satisfactory accuracy in the calculation of air temperature in the experimental room.

Calculation of the relative humidity of air and air velocity in ANSYS Fluent showed a difference in calculated and experimental data of not more than $9.5 \%$.

\section{Conclusions}

A mathematical model of heat and mass transfer is developed that describes the formation of a microclimate in a non-production building that takes into account all the main features of the object (people in the room, reducing the radiative heat exchange of air and the actual air exchange). The adequacy of the mathematical model of the microclimate was proved by comparing the calculation results in the ANSYS Fluent with experimental data.

\section{References}

1. V.A. Arutyunov, V.V. Bukhmirov, S.A. Krupennikov, Mathematical modeling of thermal operation of industrial furnaces (Metallurgy, Moscow, 1990) [In Russian]

2. Yu.A. Tabunshchikov, M.M. Brodach, Mathematical modeling and optimization of thermal efficiency of buildings (ABOK-PRESS, Moscow, 2002) [In Russian]

3. ANSYS Fluent User's Guide. Release 15.0 (2013)

4. V.V. Bakhmirov, Reference materials for solving problems on the course "FHeat and Mass Transfer" (ISPU, Ivanovo, 2009) [In Russian]

5. A.P. Laptev, S.A. Polievsky, Hygiene (Physical Culture and Sports, Moscow, 1990) [In Russian]

6. SP 118.13330.2012 Public buildings and facilities (FAU Federal State Administration, Moscow, 2014) [In Russian]

7. A.A. Yun, B.A. Krylov, Calculation and modeling of turbulent flows with heat exchange, mixing, chemical reactions and two-phase flows in the FASTEST-3D software package (Publishing house MAI, Moscow, 2007) [In Russian]

8. V.V. Bukhmirov, MATEC Web Conf. 92 (2017) 